\title{
ANALYSIS OF ROCK MASS DESTRUCTION PROCESSES WITH DIFFERENT ACTIVITY DIAGRAMS
}

\author{
Wiesław PIWOWARSKI ${ }^{1)}$, Piotr STRZAŁKOWSKI ${ }^{2)}$ and Roman ŚCIGAŁA ${ }^{2)}$ * \\ 1) AGH University of Science and Technology, ul. Mickiewicza 30, 30-059 Krakow, Poland \\ ${ }^{2)}$ Silesian University of Technology, ul. Akademicka 2a, 44-100 Gliwice, Poland \\ *Corresponding author's e-mail: roman.scigala@polsl.pl
}

\begin{tabular}{l} 
ARTICLE INFO \\
\hline Article history: \\
Received 29 June 2016 \\
Accepted 14 September 2016 \\
Available online 3 October 2016 \\
\hline
\end{tabular}

\section{Keywords:}

Survey of post-mining deformations

Mining tremors

Predictions of post-mining deformations

Wavelet transform

\begin{abstract}
The paper presents the considerations devoted to homomorphism analysis of processes: displacement and medium (rock) vibration, generating physical risk for the area of topological transformations. The aim of the study was to associate interactions: mining induced vibrations and displacement and their temporal relations. The basis for the analysis were both: sets of observed displacements - measured with GPS technology and recordings of acceleration process by using 3D vibration sensors, as well as mathematical modelling of these processes. It has been indicated, that the carrier of a formal process will be the probability space - which implies the stochastic nature of the phenomenon. Measures of the destruction occurrence - a critical process - there are the probability measures. In the estimated moment of time t, the results of observations of both processes - which are sets of real numbers - indicate that objects that are at risk of event are subjected to danger or failure. Fundamental here are mappings, used for modelling of concurrent processes, those communicate with each other. Features of timing relationships are: the structure and dynamics of processes.

The emergence of the conflict - the occurrence of a critical event - has been estimated on the basis of the lemma for reaching of the outer extremes, in terms of equivalence relations in a fixed set of circumstances. The structural similarity between the two processes, their predictability and determination of characteristic frequencies was performed with using the relevant statistics, including the so-called Hurst exponent. An important element of proceeding the threats is the relationship in the domain of extreme events as a decisive influence - the difference compared to traditional analysis. The application layer contains specific examples of estimating the risk of a critical event arising.
\end{abstract}

\section{INTRODUCTION}

Underground extraction generates - in the destruction meaning - the processes: rock mass deformation and seismic activity. They represent significant threats of environmental nature. Deformation processes are slowly progressing; they are analytically predictable in terms of time, location and measurable parameters. In the mathematical formulation, the displacement $u(x,$.$) is treated as$ a series of solutions to boundary problems of mathematical models (Evans, 2002; Piwowarski, 2000).

Seismic processes manifested by the presence of rock mass tremors, resulting in vibrations of certain sub-region, are short-lived and have a random character. Hence the paraseismic threats are characterized mainly by the probability measures.

One of the main objectives of the study was an attempt to establish the "relationship" between the sets of displacements and vibrations, definable in mathematical structures. The concern here is the possibility of measuring the size of such sets (Ucinski, 2005). The work concerns the analysis of the physical space structure, where sub-regions, as a result of mining impact, are not stable. A stochastic model characterized by the probability of a critical event will be considered, as a realization of the system in the state $\alpha$ at the time t. The probabilities of configuration changes to the system do not depend here on the whole trajectory, but only on the current state of the process. Considerations have been enhanced by using measurement results of both processes.

\section{FORMULATION OF THE PROBLEM}

The paper concerns issues relevant to the analysis of the mechanisms of wave movements and displacements in materials of properties and geometry similar to those found in the rock mass exposed to underground mining. The destruction process of the part of the rock mass layers in the vicinity of extracted deposit causes deformations and topological transformations of successive layers, often generates 
also mining tremors - which is related mainly to excess of the rock medium limit states.

For identification of limit state, the so called generalized limit function $g(X)$ is defined (Piwowarski et al., 2004), determined on random parameters. It is defined that:

$$
g(X):=\left\{\begin{array}{l}
>0 \Rightarrow \text { safe sub-region } \\
\leq 0 \Rightarrow \text { limit state }
\end{array}\right.
$$

where: $X$ - the space of parameters' state;

$$
x \in X ; \operatorname{dim} X=2
$$

So, two states of rock medium transformation are distinguished:

- $\quad$ the state of deformed medium:

$$
\Omega_{b}=\{x: g(x)>0\}
$$

- the state of singular event (occurrence of the limit state)

$\Omega_{a}=\{x: g(x) \leq 0\}$

Important here is a measurement information regarding the rock medium transformation and definition of the parameters and measures characterizing the anisotropy of its structures. Formal arrangement consists of an infinite half-plane with the edge of defined linear density, placed in the harmonic potential. In such arrangement, bulk waves may propagate, and due to presence of the surface (in this case one-dimensional), the surface waves too. In general, for the frequency and wave vector component, parallel to the surface lying outside the volume bands, one gets "real" surface waves, while within the band we can get surface resonances (leaky waves). This work studies the dynamics of the land surface due to underground mining in a threedimensional continuous medium (Daubechies, 1992). An attempt was made to describe the surface waves components of the wave vector and the process of displacement in terms of the application of the results in the description.

The dynamics of the system, consisting of halfinfinite medium limited by the surface, is described by the equations of medium motion, supplemented with boundary conditions expressing the equations of motion of the surface layer. Solutions for the real frequencies without external disturbances are referred to as surface states or surface waves. If they are outside the volume bands, i.e. have all partial waves declining - they are called true surface waves, as they actually refer to the surface area.

On the other hand, irregular trajectories of the displacement process are analyzed here as mean values. Function $M: I \times I \rightarrow R$ is called the mean function if:

$\min (x, y) \leq M(x, y) \leq \max (x, y), \quad x, y \in I$
Typically, variables occurring here are not constant over time, moreover, the loads acting inside the sub-region also change over time.

\section{THE MODEL OF ANALYSED PROCESS}

The simplest two-dimensional system, in which there may surface waves occur, is a half-infinite elastic surface bounded by straight edge. To simplify the notation we choose a coordinate system such that the "surface" is lying in the plane $(x, y)$ and its edge is defined by $\mathrm{x}$-axis. The equation of motion for such medium with deflections $\mathrm{u}(\mathrm{x}, \mathrm{y} ; \mathrm{t})$ perpendicular to the plane, i.e. transverse movements out of the plane (Evans, 2002) is:

$$
\begin{aligned}
& \frac{\partial^{2} u(x, y ; t)}{\partial t^{2}}=c^{2}\left[\frac{\partial^{2} u(x, y)}{\partial x^{2}}+\frac{\partial^{2} u(x, y)}{\partial y^{2}}\right] \\
& (x, t) \in R^{2}, x \in R \\
& 0<y<\infty \\
& u(x, 0)=f(x) \\
& u_{t}(x, 0)=g(x)
\end{aligned}
$$

Problem (5) has exactly one solution of class $C^{2}$, namely:

$u(x, t)=\frac{1}{2} f(x+c \cdot t)+\frac{1}{2} f(x-c \cdot t)+\frac{1}{2 c} \int_{x-c t}^{x+c \cdot t} g(\tau) d \tau$

where: $u(x, t))$ - non-stationary process,

$$
\begin{aligned}
& f \in R \text { of class } C^{2} \\
& g: R \rightarrow R \text { of class } C^{1} \\
& c \text { - parameter, } \\
& (x+c \cdot t, x-c \cdot t) \in R^{2} \text { - linear isomorphism }
\end{aligned}
$$

Using the Sturm-Liouville definition (Evans, 2002) for the problem (6), it suggests the following solution (7) - the wave motion:

$u(x, t)=\sum_{k=1}^{\infty} a_{k} \cdot \sin k x \cdot \cos k t$

in addition:

$a_{k}=\frac{2}{\pi} \cdot \int_{0}^{\pi} f(x) \cdot \sin k x d x$

In real conditions, wave motion is subjected to damping. The introduction of damping for all degrees of freedom formally corresponds to the immersion of the entire system in the liquid of a certain viscosity. Such operation allows showing, in the local density of states, the peaks of a Dirac delta in the form of narrow Lorentz's maxima. In fact, every multi-body system manifests certain energy losses. After the introduction of a member responsible for damping, the equation of motion and the boundary condition for the specified system, leads to the following description (8): 


$$
\begin{aligned}
& \frac{\partial^{2} u(x, y ; t)}{\partial t^{2}}= \\
& =c^{2} \cdot \frac{\partial^{2} u(x, y ; t)}{\partial y^{2}}-c \cdot k_{1}^{2} \cdot u(x, y ; t)-\gamma \cdot \frac{\partial u(x, y ; t)}{\partial t}
\end{aligned}
$$

or with more general form:

$$
\rho_{S} \frac{\partial^{2} u(x, y ; t)}{\partial t^{2}}=T\left(\frac{\partial u}{\partial y}\right)_{y=0}-\beta \cdot u-\gamma \cdot \frac{\partial u(x, y ; t)}{\partial t}
$$

where: $x, y$ - space coordinates; $(\mathrm{x}, \mathrm{y}) \in$ top $\mathrm{R}^{2}$, $t$ - time coordinate,

$\mathrm{x}, \mathrm{y} ; \mathrm{t}) \in$ top $\mathrm{R}^{2+1}$,

$c, k_{1}, \beta, \gamma, \rho_{s}$ - parameters,

$T$ - differential operator,

top - topology.

Wave equation in the dimensions $\mathrm{n}=1,2,3$ has the solution respectively as: d'Alembert's, Poisson's and Kirchhoff's (Evans, 2002). The existence and uniqueness of solutions are defined by the Huygens principle. Inhomogeneous wave equation is proceeded by the Duhamel method. The variables and loads acting inside the sub-region change over time, the variation reveals the characteristics of medium randomness and requires different methods of analysis - the use of random functions.

Let $X(t)$ - stochastic process. Particularly interesting and useful here is the variance analysis of stochastic process (Piwowarski et al., 2015). In classical terms, we have:

$$
\operatorname{Var}[X(t)]=E\left[X^{2}(t)-E\left(X^{2}(t)\right)\right]
$$

For the asymptotic states of (9), we have:

$$
\left\langle X^{2}(t)\right\rangle=\left\{\begin{array}{l}
|t|-\text { Wiener process } \\
|t|^{\alpha}-\text { anomalous diffusion } \\
\infty \quad \text { Levy process }
\end{array}\right.
$$

Variance (10) scales asymptotically as follows:

$$
\begin{aligned}
& \left\langle X^{2}(c t)\right\rangle=c^{\alpha}\left\langle X^{2}(t)\right\rangle \\
& (c, \alpha)>0
\end{aligned}
$$

The analysis of the real process based only on the variance of the stochastic process is not sufficient enough - the realizations of a stochastic process should be analyzed in a broader sense.

\section{NUMERICAL PROCEDURE}

The optimization of observations of the processes with spatiotemporal dynamics is a nontrivial problem. In order to reduce the correlation between various sensors recording the characteristic properties of the process, the information matrix of the member describing the correlations was employed (Brockwell and Davis, 1996). Fisher information matrix (Brockwell and Davis, 1996; Zieliński, 2007) has the following form here:

$$
F_{M}\left(x^{1}, x^{2}, \ldots, x^{n}\right)=\sum_{i=1}^{n} \sum_{j=1}^{n} \int_{T} w_{i j}(t) g\left(x^{i}, t\right) g^{T}\left(x^{j}, t\right) d t
$$

where:

$$
\begin{aligned}
& g(x, t)=\left[\frac{\partial y(x, t ; \theta)}{\partial \theta_{1}}, \ldots, \frac{\partial y(x, t ; \theta)}{\partial \theta_{n}}\right]_{\theta=\theta^{0}}^{T} \\
& W(t)=w_{i j}(t)=C^{-1}(t) \\
& C(t)=g\left(x^{i}, x^{j} ; t\right) \\
& C(t) \quad \text { - covariance matrix, } \\
& n \quad \text { - number of observations. }
\end{aligned}
$$

If the covariance matrix depends on the distances between sensors, one has:

$g\left(x^{i}, x^{j} ; t\right)=\sigma^{2} \cdot e^{\left(-\frac{\left\|x^{i}-x^{j}\right\|}{\beta}\right)}$

where: $\beta$ - coefficient determining the correlation of observations

Large $\beta$ values imply strong correlation of observations.

For one-dimensional system without dumping, equation (8) takes the form:

$\frac{\partial^{2} u(t, x)}{\partial t^{2}}-c \cdot \frac{\partial^{2} u(t, x)}{\partial x^{2}}=0$

The sketch of numerical solution of the equations (8) or (17).

We define the operator $L(.,$.$) , which, acting on$ the function $u(x, t)$, generates certain differential expřession, which can be represented by second order partial derivatives with respect to time and secondorder with respect to position.

$$
\left.\begin{array}{rl}
L u(x, t)= & \frac{u(x+h, t)-2 u(x, t)+u(x-h, t)}{h^{2}} \\
u(x, t)= & \frac{\partial^{2} u(x, t)}{\partial x^{2}}-\frac{\partial^{2} u\left(x, t+\frac{k}{2}\right)}{\partial x^{2}}+ \\
& +\frac{\partial^{2} u\left(x, t-\frac{k}{2}\right)}{\partial x^{2}}+O\left(h^{2}+k^{2}\right)
\end{array}\right\}
$$

Numerical solution bases on the decomposition of the region of interest and the use of partial differential algorithm defined over smaller subregions (Cholewa and Dłotko, 2000). Elimination of the unknowns is carried out within the sub-regions. In two dimensions the analyzed areas in general are polygons. The displacement process for small observation steps has been modelled by the time series. Mapping of the process as a time series (Brockwell and Davis, 1996) is defined as follows: 
Point $1_{\oplus}$

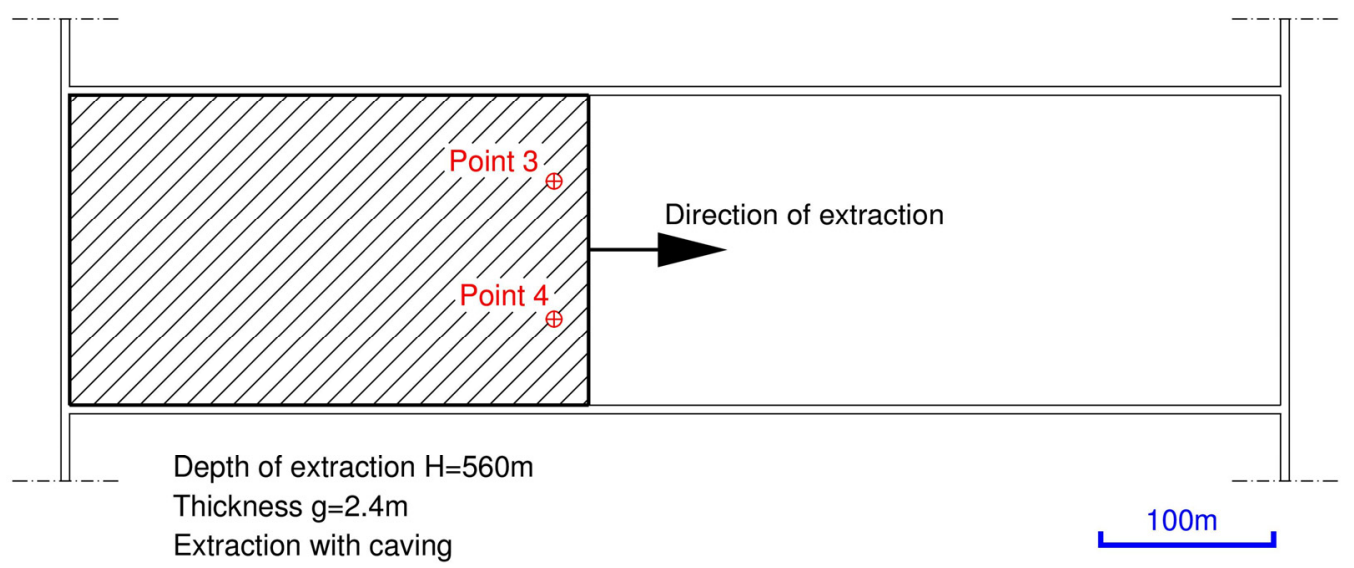

Point $2_{\oplus}$

Fig. 1 Location of survey stations against mining extraction field at the moment of analysed tremor occurrence.

$Y^{M}(t)=a_{0}+\sum_{i=1}^{n / 2}\left[\begin{array}{l}a_{i} \cdot \sin \left(\frac{2 \pi}{n} \cdot i \cdot t\right)+ \\ +b_{i} \cdot \cos \left(\frac{2 \pi}{n} \cdot i \cdot t\right)\end{array}\right]$

Model parameters are estimated with the following procedure:

$$
\begin{aligned}
& \begin{array}{c}
a_{0}=\frac{1}{N} \sum_{i=1}^{n} Y\left(t_{i}\right) \\
a_{i}=\frac{2}{N} \sum_{i=1}^{n} Y\left(t_{i}\right) \cdot \sin \left(\frac{2 \pi}{n} \cdot i \cdot t\right) i=1,2, \ldots,(n / 2)-1
\end{array} \\
& b_{i}=\frac{2}{N} \sum_{i=1}^{n} Y\left(t_{i}\right) \cdot \cos \left(\frac{2 \pi}{n} \cdot i \cdot t\right) i=1,2, \ldots,(n / 2)-1
\end{aligned}
$$

\section{COMMUTATION OF COLLECTIVE DEFORMATION PROCESSES: MINING INDUCED VIBRATIONS AND DISPLACEMENTS}

At given point locations of active mining area, continuous survey of displacements $\{U\}$ and vibrations $\{\mathrm{D}\}$ is conducted:

- $U=\left(u_{1}, u_{2}, \ldots, u_{n}\right)$ - the set of displacements registered at given point location in determined moments of time: $t_{1} \supset u_{1}, t_{2} \supset u_{2}, \ldots, t_{n} \supset u_{n}$ (GPS survey),

- $D=\left(d_{1}, d_{2}, \ldots, d_{m}\right)$ - the set of registered vibrations at the same point location in determined moments of time (three-dimensional vibration sensors),

- These processes have some specific properties:

- displacement process $U \supset u(x, t)$ is continuous, extensive in time,
- vibration process $D \supset d(x, t)$ is singular and of short duration,

- $\quad$ both processes begin and end at different times (asynchronous).

The survey results analyses (vibrations displacements) show that quasi-relation exists between registered sets $\{U\} \rightarrow\{D\}$, namely:

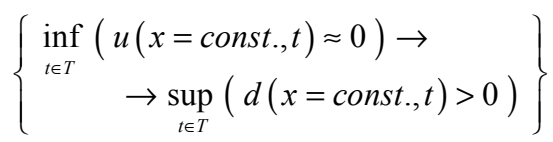

for relation $(*)$, process $d(t, x)>0$ occurs with high probability. The relation $\left(^{*}\right)$ is confirmed by exemplary results of displacements and vibration monitoring results presented in Figure 2.

The surveys were conducted in the area of underground mining extraction influences. Mining works were led with longwall system (Fig. 1). Four ground surface survey stations (points) were used with different horizontal locations against mining field, according to Figure 1. The trajectories of the observed point No. 4, in the $\mathrm{x}, \mathrm{y}, \mathrm{z}$ directions are shown in Figures 2 a-c. The time coordinate of the tremor occurrence has been presented on the background of displacement distribution over time. It was possible to overlay such survey information by placing the GPS station and 3-dimensional vibration sensor in the same location.

The measurements were conducted in a quasicontinuous (frequency $50 \mathrm{~Hz}$ ), specially prepared stations, equipped with a GPS device and a vibration 


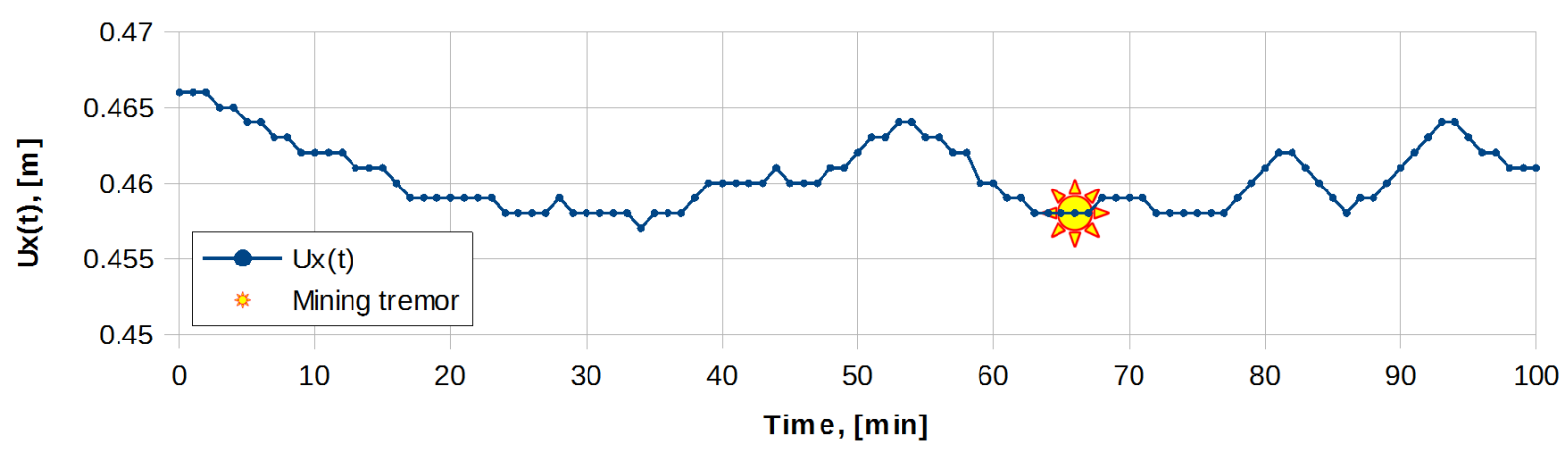

Fig. 2a Trajectory $\mathrm{U}_{\mathrm{X}}(\mathrm{t})$ for point No. 4; time window $\Delta T=100$ [min] $\Delta t=1$ [min].

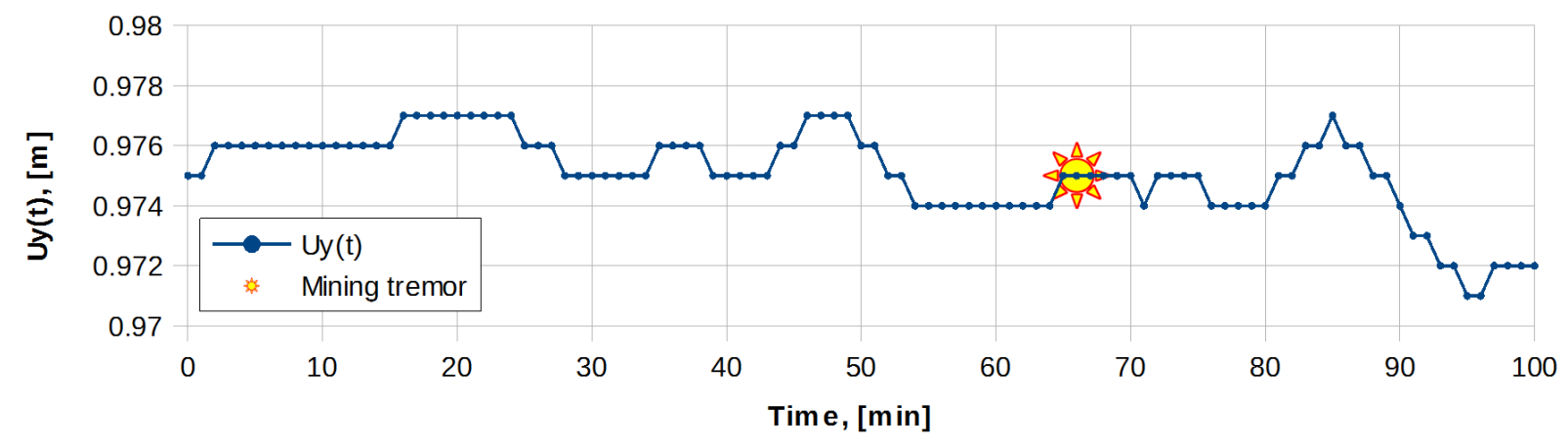

Fig. 2b Trajectory $\mathrm{U}_{\mathrm{y}}(\mathrm{t})$ for point No. 4; time window $\Delta T=105$ [min] $\Delta t=1$ [min].

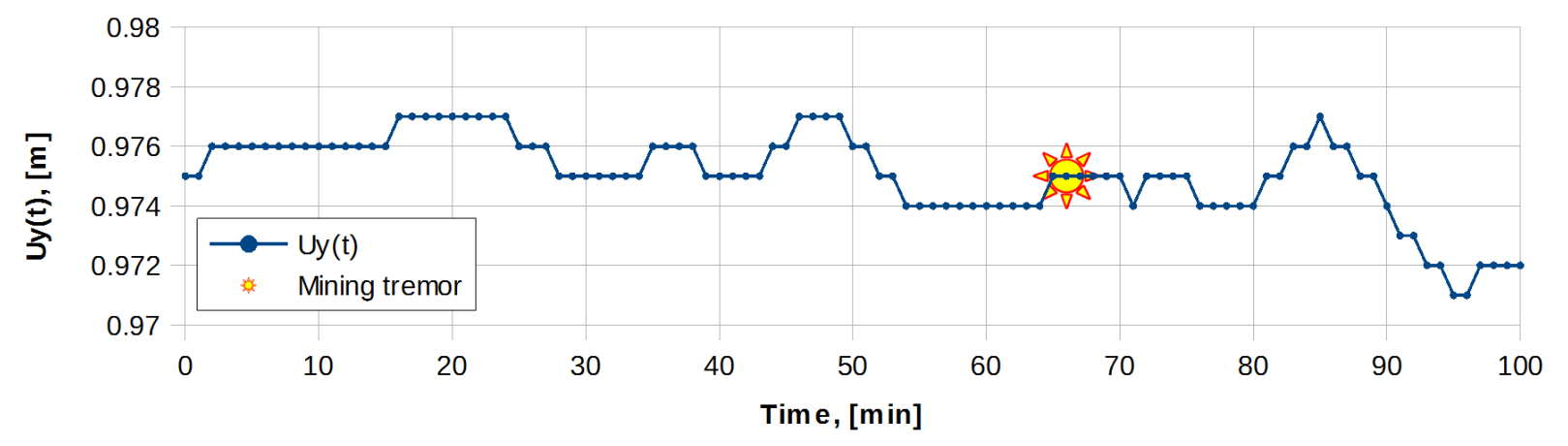

Fig. 2c Trajectory $\mathrm{W}(\mathrm{t})$ for point No. 4; time window $\Delta T=105[\mathrm{~min}] \Delta t=1[\mathrm{~min}]$.

sensor. The development of GPS satellite observations had been worked for the high precision geodetic networks, through a link to the European Terrestrial Reference System ETRF89 (Narkiewicz, 2003).

Obtained results confirm that the minimum of displacement gradient $\nabla_{x} u(x, t) \approx 0$ is observed at the moment of time very close to the mining tremor occurrence (Sokoła-Szewioła, 2011).

In Figure 3 the results of subsidence (vertical displacement) modelling have been shown, related to survey results.

\section{FREQUENCY DOMAIN CHARACTERISTIC OF THE PROCESS}

It is an adequate definition of the artificial representation of the registered process. Frequency analysis has a good physical interpretation and solid theoretical background. The Fourier transform states, that the spectrum of such signals changes over time and one obtains averaged results (in the analysis window) - this approach is often not sufficient enough. Using the digital recording of rock mass tremors, the self-similarity of vibration process in unsteady displacement phase by means of $R / S$ statistics graphs over time has been determined (Daubechies, 1992; Hurst, 1951). The statistics mentioned above is characterized more thoroughly in the next section of the paper. Obtained here value of parameter $\mathrm{H}$ (Hurst) serves for the qualitative analysis of the event flow. For the interpretation purposes, the graphs of $\mathrm{R} / \mathrm{S}$ in the time function are subjected to linearization in the double logarithmic coordinate 


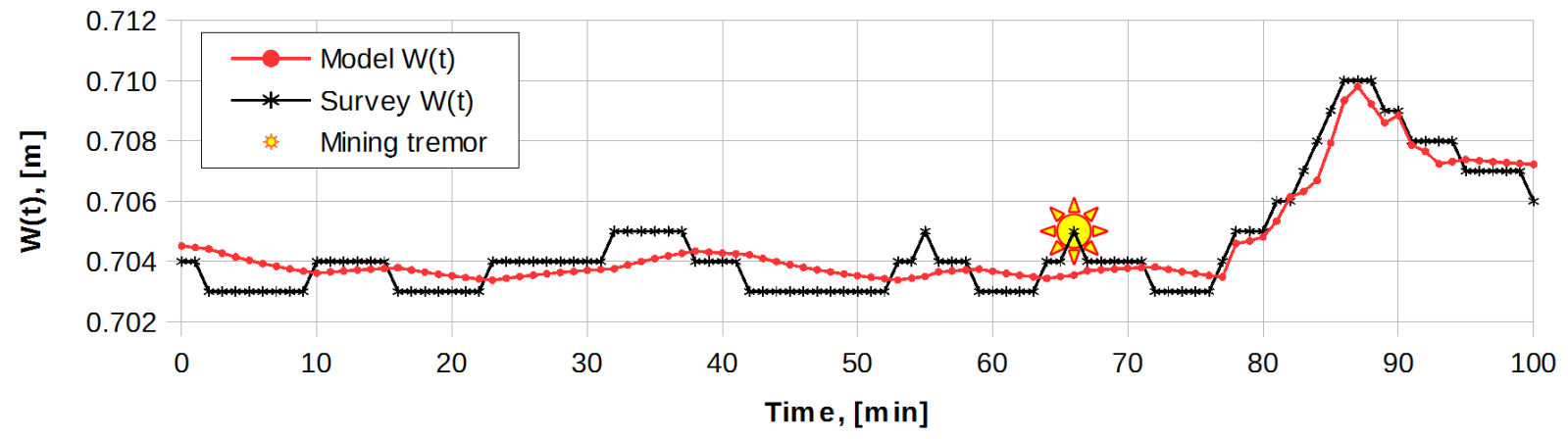

Fig. 3 The comparison of trajectories $\mathrm{W}(\mathrm{t})$ obtained on the basis of surveys and theoretical modelling (time series). Point No. 4; time windows $\Delta T=105$ [min] $\Delta t=1[\mathrm{~min}]$.

Vertical Displacement W(t). Point No 4, 9.10.2010. Hurst Exponent

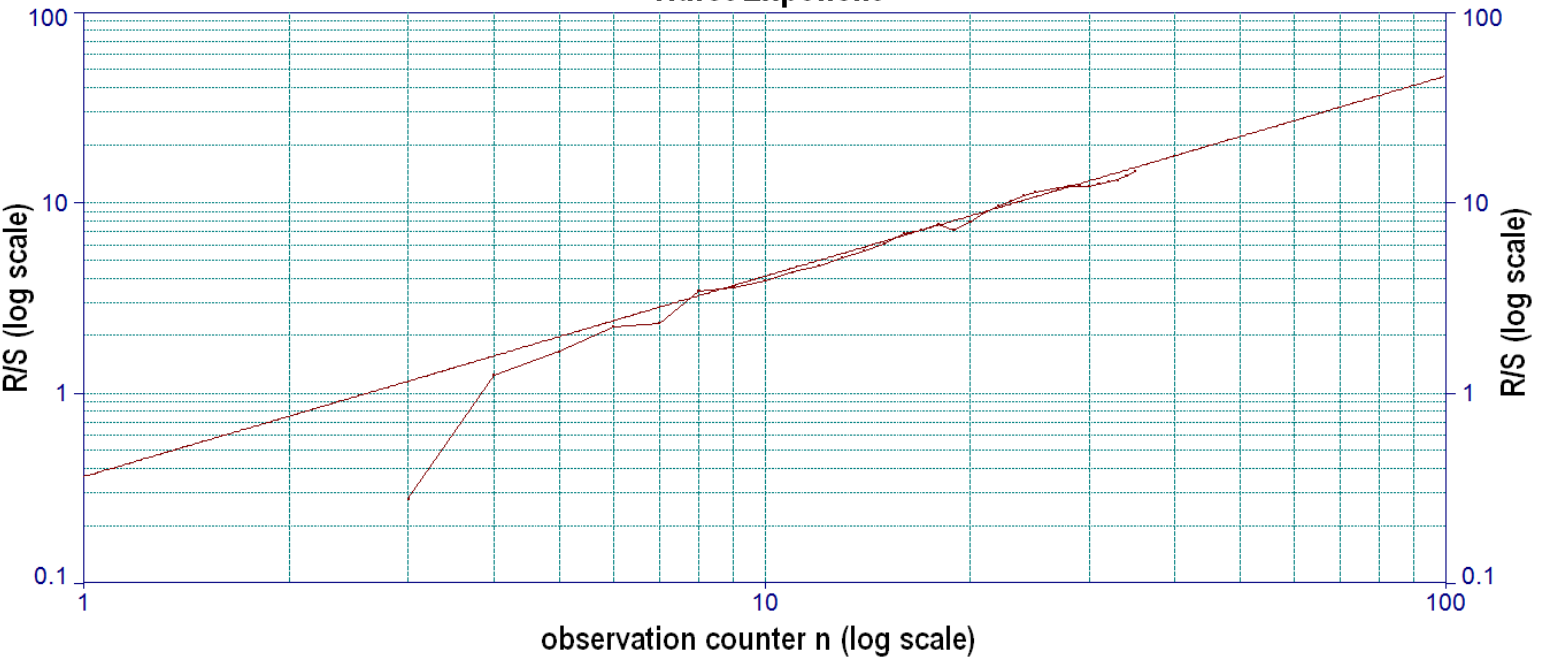

Fig. 4 The graph of $(\mathrm{R} / \mathrm{S})$ over time for vertical displacement. Point No. 4, time window $\Delta T=105$ [min], $\Delta t=1[\mathrm{~min}], 9.10 .2010$.

system to give a linear relationship with a slope of a straight line $\mathrm{H}$ :

$\log \frac{R(n)}{S(n)} \approx H \cdot \log (n)+c$, c-constant

The projection of the statistics $\mathrm{R} / \mathrm{S}$ as well as wavelet transform of displacement process - Figures 4 and 5 - have been performed for time window $\Delta T$, by using dedicated software (AutoSignal, 2003). The trajectory of the process of vertical displacements reveals no significant frequencies of process fluctuations - Figure 5.

\section{MINING TREMORS}

Critical phenomena that take place at different areas often show a similarity in both its course and the way of developing. In mining conditions, seismic events are usually anthropogenic in nature - caused by underground mining works.

Post-mining processes that occur quickly or are short-lived, are subjected to the methods of monitoring or experiment (cave-in, rock bursts ...). On the other hand, processes which are slow in their nature - displacements of the medium, are subjected to both observation and modelling. Most of important technical problems from the field of the rock mass structures deformation remain unresolved. These problems involve a wide range of scales, which are not amenable to a single definition. The combination of the simulation tools directly with measuring devices to control the real-time computer simulation of processes and representations is very important from the utilitarian point of view (Lasocki and Orlecka-Sikora, 2008). Researches in this field refer to the applications of so called Dynamic Data Driven Applications Systems (DDDAS) (Burges, 1998; Januszewski, 2006).

Surveys of dynamic phenomena show, that blocky cracking of rock layers and their movement toward extracted area usually generates mining tremors. In addition, at the surface level rapidly vanishing local subsidence areas are observed (Kazimierczyk, 1991). 


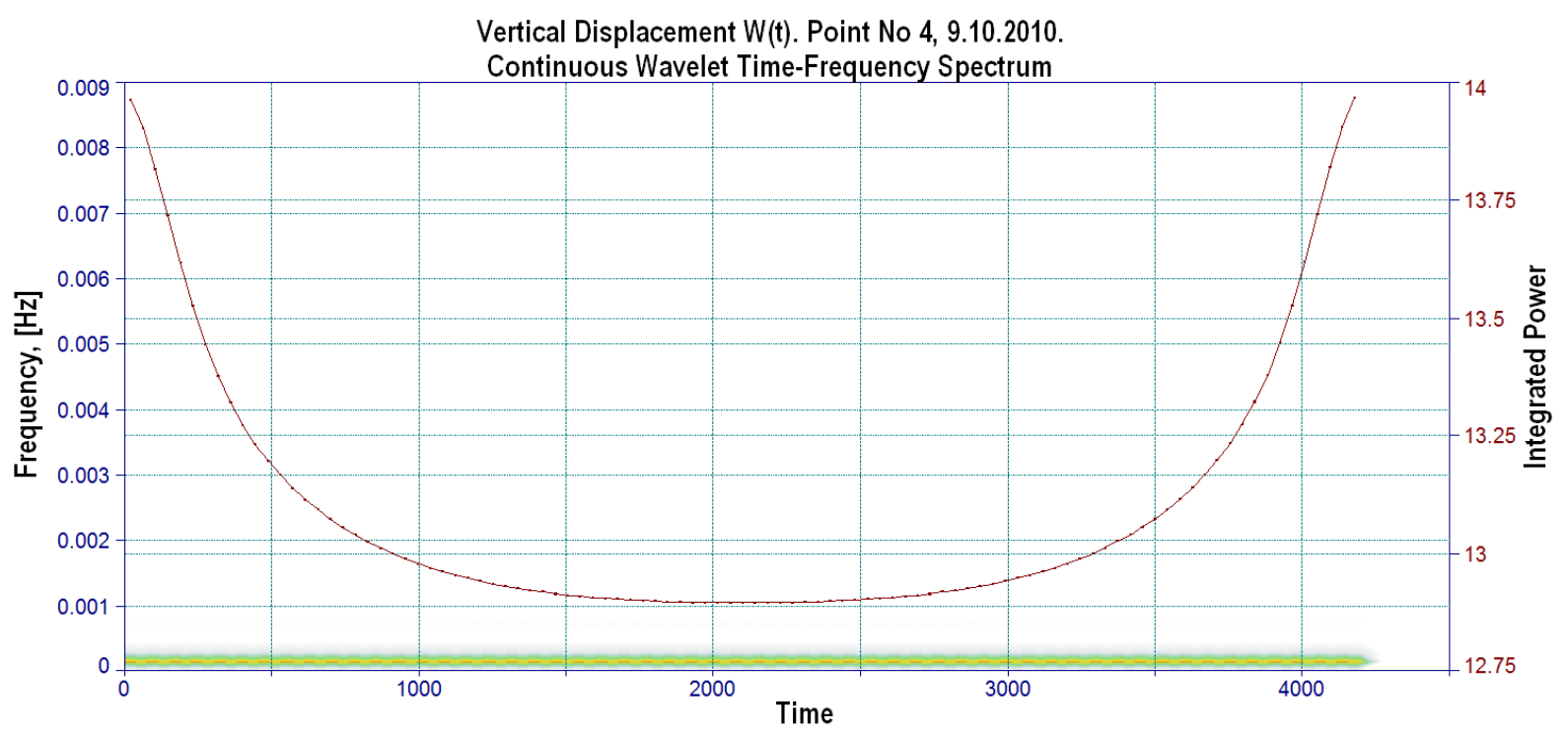

Fig. 5 The graph of wavelet transform of vertical displacement over time. Point No. 4, time window $\Delta \mathrm{T}=105[\mathrm{~min}], \Delta \mathrm{t}=1[\mathrm{~min}], 9.10 .2010$.

In Polish mining basins, the origins and nature of the mining tremors are different. So the parameters characterizing the propagation of seismic waves are inconsistent even for given basin. For example, in LGOM area, the dispersion of registered vibrations acceleration is $\pm 230 \%$ (Kazimierczyk, 2001).

Classical analysis generally does not allow to estimate whether the nature of the process is purely random (no causal mechanisms), or the process is hidden, with internal non-linear dynamics, and the results obtained present deterministic chaos. Evaluation of the type of irregularities and separation of statistical ranges of measurement dispersion and chaotic course of the process is of utilitarian importance. Hurst exponent allows evaluating the nature of the recorded data - determining the correlation of measured data.

For assessment of the recorded data nature, statistical procedures have been employed, namely the calculation of the Hurst exponent. Hurst exponent $\mathrm{H}$ is calculated in accordance with (Hurst, 1951), on the basis of the equation (22):

$\frac{R}{S}=(a \cdot M)^{H}$

where: R - series range,

S - standard deviation of originally recorded data,

M - number of observations (cardinality),

a - constant.

$H= \begin{cases}0.5 & : \text { random process, events are uncorrelated, } \\ \in(-0 \rightarrow 0.5) & : \text { ergodic process, returning to the mean value, } \\ \in(0.5 \rightarrow 1) & : \text { process defining trend. }\end{cases}$

The nature of $\mathrm{R} / \mathrm{S}$ : re-scaled range estimates random walking as dispersions of displacement fluctuations, whereas exponent $H$ may be related to the risk value.

The tremors process has also the following properties:

- limited energy concentrated in the neighbourhood of considered point,

- mean value $=0 ; \int_{-\infty}^{\infty} \Psi(t) d t=0$,

- finite bandwidth.

The analyzed process may be represented as the sum of scaling functions and wavelets, a family of orthogonal functions, discrete wavelet transform which means that it maps accurate, undisturbed time information. The signal from measuring device was recorded in real time in $\mathrm{R}^{3}$ space. The example of acceleration course over time for survey point No. 4 is shown in Figure 6.

One of the main objectives of the model theory is the study of sets definable in mathematical structures. The concern is the possibility of measuring the size of such sets. In the case of stable structures, definable sets are measured by Morley rank. In general one can use here the concepts of topological dynamics. What can be useful here are the concepts of poorly generic sets and types, almost periodic inside definable groups. They are closely connected with the dynamic properties of certain streams associated with such groups.

\section{WAVELET TRANSFORM}

The results of the process measurement in the wavelet transform CWT (Continuous Wavelet Transform) (Daubechies, 1992) have been reconstructed by superposition of wavelets. As a result, the information obtained of one component has been divided into several bands with different 

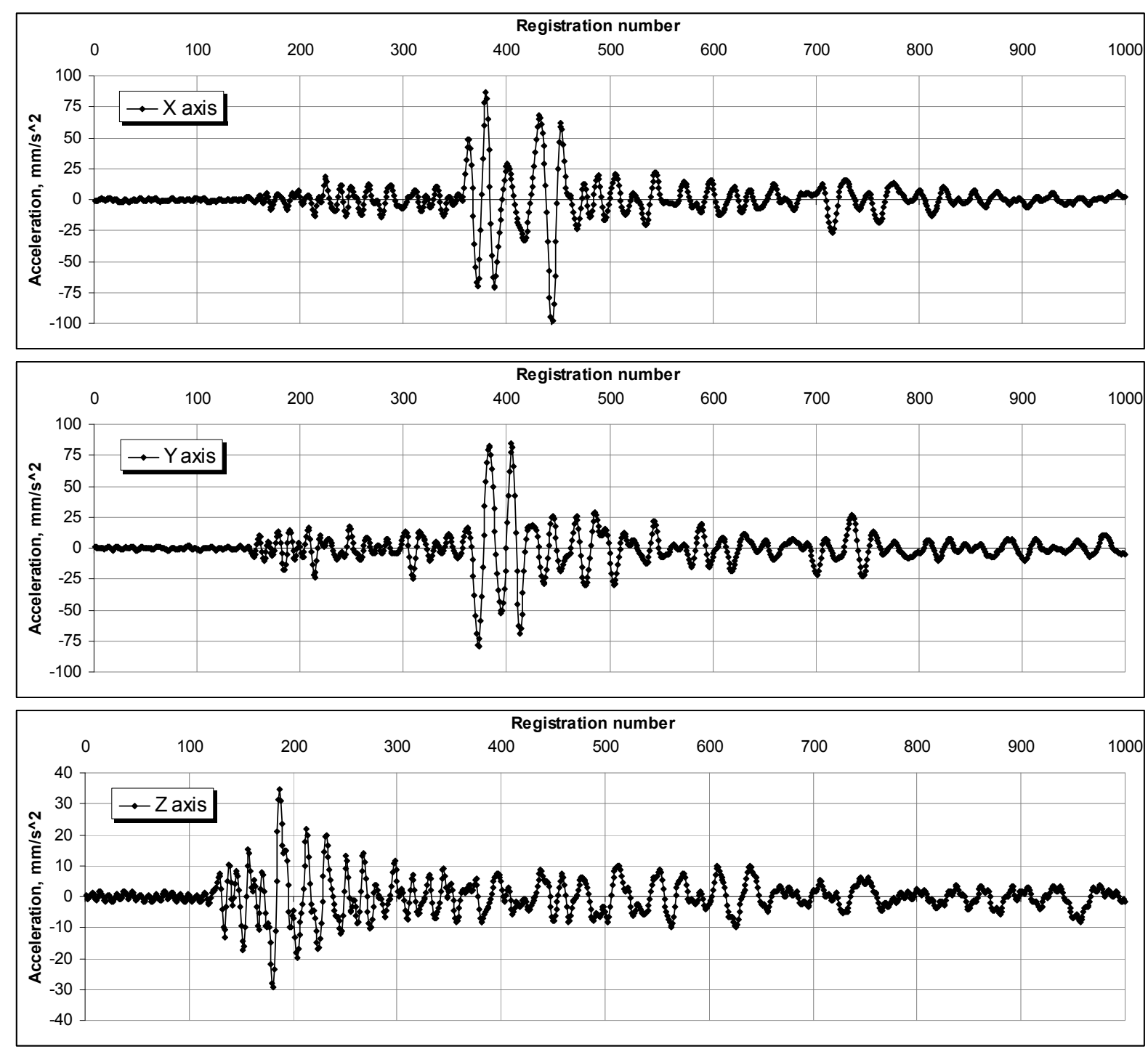

Fig. 6 Mining tremor from 9.10.2010, 17:31:38; Energy 8.6*10 $0^{4}$ [J]; Point No. 4.

frequencies, different time scale and small mutual correlation (Burges, 1998). Wavelet transform parameters are: location and its rescaling, hence this transformation allows obtaining data concerning the change of individual frequency components over time. This results in two filtered signals representing the decomposition of the input signal: low frequency $y_{\text {low }}$ and high frequency $\mathrm{y}_{\text {high }}$ signals. Time-dependent features are the structure and dynamics. The structure defines which events are premises in relation to others, being their consequences. The dynamics determines the moments of time, when dependencies are implemented. The analyzing function $w\left(\frac{t-b}{a}\right)$, by using the scale parameter, expands or contracts depending on the frequency. Wavelet transform is a two-dimensional function, which uses convolution of the signal $\mathrm{x}(\mathrm{t})$ with analyzing function (Hurst, 1951):
$C(a, b)=$

$=x(t) \cdot\left[|a|^{-0.5} \cdot w\left(\frac{t}{a}\right)=|a|^{-0.5} \cdot \int_{-\infty}^{\infty}-\left(\frac{t-b}{a}\right) x(t) d t\right]$

b - translation parameter, a - scale parameter; $-\infty<a<\infty$.

$w\left(\frac{t}{a}\right)=\frac{1}{\sqrt{2 \sigma}} \cdot \exp \left(-\frac{t^{2}}{2 a^{2}} \cdot \cos \left(\frac{p t}{a}\right)\right) ; p \sim 5$

Wavelet analysis is a method for analyses of non-stationary signals, where frequency spectrum changes over time. Time - frequency methods point, which frequency component is the strongest one at given moment. Performed here spectral analysis of the registered process of mining induced vibrations allowed to identify specific harmonics - significant frequency. Registered distribution of vibration over time is "glued" together by means of wavelets superposition. The result is the separation of 


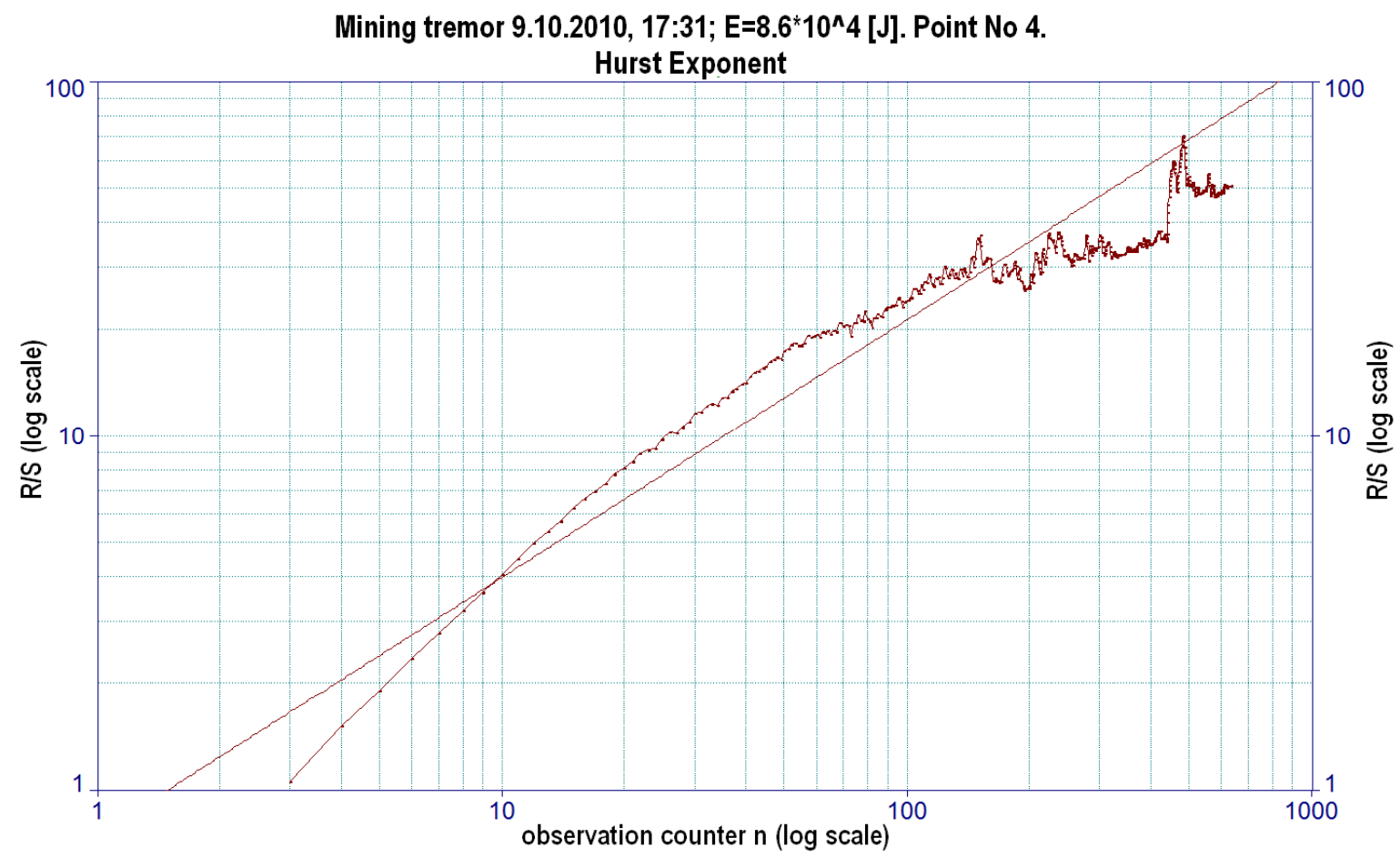

Fig. 7 The graph of $(\mathrm{R} / \mathrm{S})$ over time for registered mining tremor. Point No. 4, time window $\Delta \mathrm{T}=105$ [min], $\Delta \mathrm{t}=1[\mathrm{~min}], 9.10 .2010$.

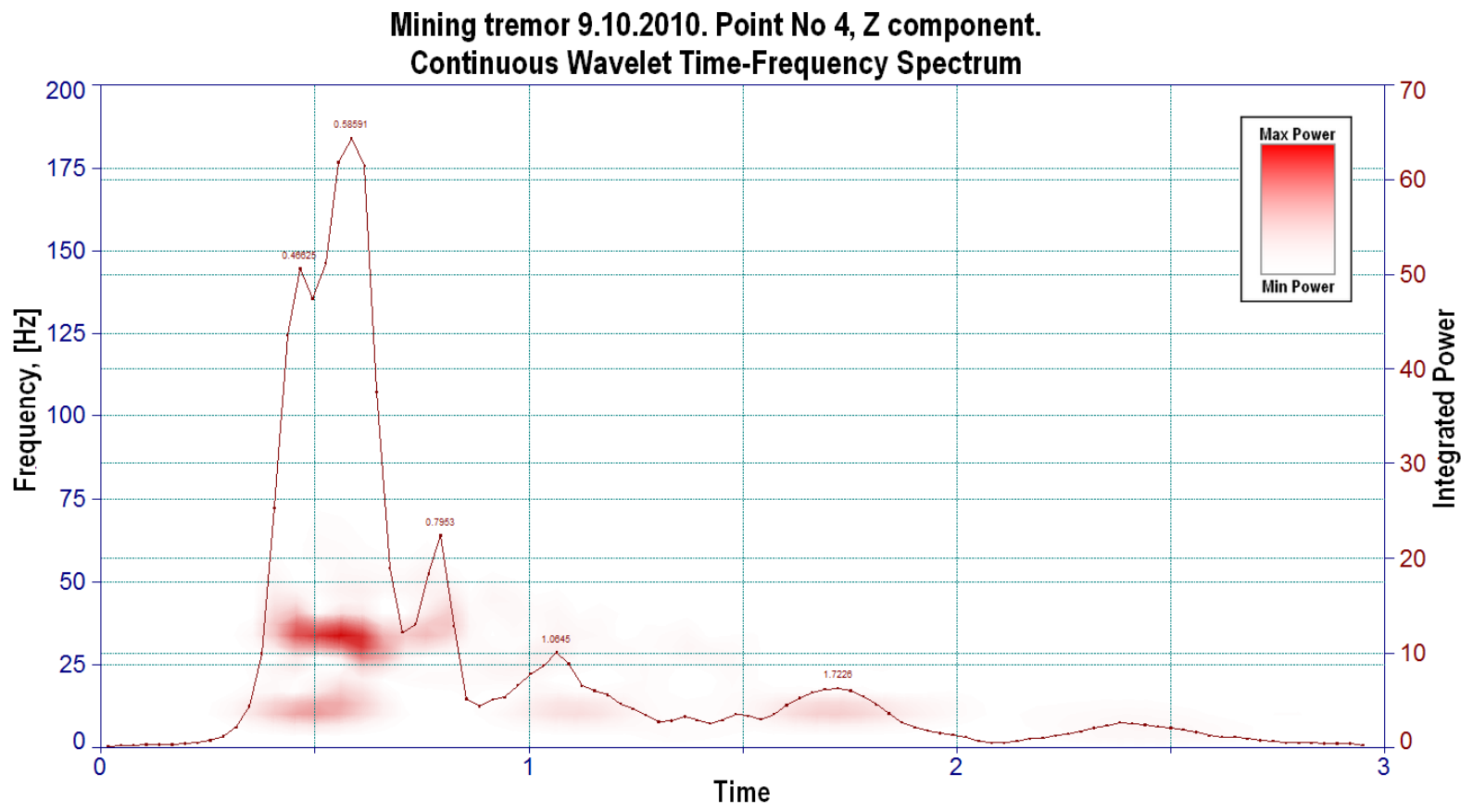

Fig. 8 The graph of wavelet transform of vertical component of registered mining tremor over time. Point No. 4 , time window $\Delta \mathrm{T}=105[\mathrm{~min}], \Delta \mathrm{t}=1[\mathrm{~min}], 9.10 .2010$.

information contained in one component into several bands of different frequencies, different time scale and small mutual correlation. Wavelet transform parameters are the position and scaling of the wavelet, hence this transformation allows obtaining of information concerning the change of individual frequency components over time.
The projection of the statistics $\mathrm{R} / \mathrm{S}$ as well as wavelet transform of mining induced vibration process - Figures 7 and 8 - have been performed for time window $\Delta T$. The trajectory of the vibrations process reveals significant frequencies of process fluctuations. 


\section{CONCLUSIONS}

Considerations and numerical experiments presented in this work make it possible to present the following conclusions:

1. The analysis of the deformation process in active mining area indicates that the dynamic local change of the medium parameters significantly affects the initiation of the singular process. The probability of critical phenomenon occurrence can be estimated here by using the algorithms of time series analysis.

2. An attempt to detect the time-structural patterns has been made in periods of the analyzed process activity. Such constructions have been used as: evaluation of irregularities and separation of statistical ranges of measurements dispersion and chaotic course of the analysed process. The discrete wavelet transform was used as well as the evaluation of the nature of the recorded data through statistical procedures - calculation of the Hurst exponent. The experimental results confirm the effectiveness of the methods used.

3. The similarity of processes was analyzed: deformations, vibrations - as an important measure from inference point of view. The similarity function allows creating semantically coherent groups. Experiments carried out on the obtained data sets indicate, that despite the correctness of geometric analysis, the processes are formally considered as of poor similarity.

4. Measuring system consisting of hardware systems: GPS and vibration sensors are the tools for the registration and transfer of data regarding medium state in the form of sets of measurements results performed in the considered area. It is, in a certain sense, the algorithm for generating information concerning the state of the monitored object - mining area.

5. The method of domain knowledge acquisition concerning the displacements of observing points - GPS technology and vibrations recording was realized through the user interfaces and properly processed computer simulations. Knowledge acquired in this way should be in interaction with the assessments of experts.

\section{REFERENCES}

AutoSignal v1.7.: 2003, Systat Software Inc., AutoSignal: Pioneering automated signal analysis and advanced filtering. Users Guide, by SeaSolve Software Inc. Framingham, USA.

Brockwell, P.J. and Davis, R.A.: 1996, Introduction to time series and forecasting. Springer - Verlag, New York Inc., $435 \mathrm{pp}$.

Burges, J.C.C.: 1998, A Tutorial on support vector machines for pattern recognition. Data Mining and Knowledge Discovery, No. 2, 121-167.
Cholewa, J.W. and Dłotko, T.: 2000, Global Attractors in Abstract Parabolic Problems. Lecture Note, Series 278, Cambridge University Press, Cambridge, 248 pp.

Daubechies, I.: 1992, Ten lectures on wavelets. CBMS-NSF Regional Conference Series in Applied Mathematics, Society for Industrial \& Applied Mathematics, Philadelphia, Pensylvania, $355 \mathrm{pp}$.

Evans, L.C.: 2002, Partial differential equations. Wydawnictwo Naukowe PWN, Warszawa, 632 pp., (in Polish).

Hurst, H.E.: 1951, Long term storage capacity of reservoirs. Transactions of American Society of Civil Engineers, 116 , Issue 1, 770-799.

Januszewski, J.: 2006, Satelite systems GPS, Galileo and others. Wydawnictwo Naukowe PWN, Warszawa, 342 pp., (in Polish).

Kazimierczyk, M.: 1991, The nature of major cracks roof rock in LGOM copper mines. Part I \& II Rudy, Metale, No 2, 52-60 \& 3, 101-107, (in Polish).

Kazimierczyk, M.: 2001, The maximum accelerations of surface vibrations in the area of LGOM. Conference „Warsztaty Górnicze”. Wydawnictwo IGSMiE PAN, Kraków, 551-559, (in Polish).

Lasocki, S. and Orlecka-Sikora, B.: 2008, Seismic hazard assessment under complex source size distribution of mining-induced seismicity. Tectonophysics, 456, Issues 1-2, 28-37. DOI: $10.1016 /$ j.tecto.2006.08.013

Narkiewicz, J.: 2003, Global positioning system. Structure, operation, application. Wydawnictwa Komunikacji i Łączności, Warszawa, 164 pp., (in Polish).

Piwowarski, W.: 2000, Prediction model of a non-stationary post-mining dislocation process, verified by means of geodetic surveys. PIGiK, 47, Issue 100, Warszawa, 183-198, (in Polish).

Piwowarski, W., Fajklewicz, Z., Radomiński, J., Stewarski E. and Tajduś, A.: 2004, Rock mass deformation changes research for restoration of building ground value. Wydawnictwo AGH, Kraków, 244 pp., (in Polish).

Piwowarski, W., Isakow, Z. and Juzwa, J.: 2015, Estimation of the mining damage risk in the hypothetical impact area of the concurrent processes of rock mass disorders. Archives of Mining Sciences, 60, Issue 4, Kraków, 889-903. DOI: 10.1515/amsc-2015-0058

Sokoła-Szewioła, V.: 2011, Research studies and modelling of the changing in time vertical displacements of the mining area in time at the period of tremor occurrence induced by longwall mining. Wydawnictwo Politechniki Śląskiej, monograph No 326, Gliwice, 173 pp., (in Polish).

Ucinski, D.: 2005, Optimal measurement methods for distributed-parameter system identification. CRC Press, 339 pp.

Zieliński, P.: 2007, Digital signals processing: from theory to applications. Wydawnictwa Komunikacji i Łączności, Warszawa, 830 pp., (in Polish). 